\title{
Visual and haptic influence on perception of stimulus size
}

\author{
GEORGE H. VAN DOORN, BARRY L. RichaRdSON, \\ Dianne B. Wuillemin, ANd Mark A. Symmons \\ Monash University, Churchill, Victoria, Australia
}

\begin{abstract}
In six experiments, subjects judged the sizes of squares that were presented visually and/or haptically, in unimodal or bimodal conditions. We were interested in which mode most affected size judgments in the bimodal condition when the squares presented to each mode actually differed in size. Three factors varied: whether haptic exploration was passive or active, whether the choice set from which the subjects selected their responses was visual or haptic, and whether cutaneous information was provided in addition to kinesthetic information. To match the task for each mode, visual presentations consisted of a cursor that moved along a square pathway to correspond to the haptic experience of successive segments revealed during exploration. We found that the visual influence on size judgments was greater than the influence of haptics when the haptic experience involved only kinesthesis, passive movement, and a visual choice set. However, when cutaneous input was added to kinesthetic information, size judgments were most influenced by the haptic mode. The results support hypotheses of sensory integration, rather than capture of one sense by the other.
\end{abstract}

This report will consider sensory integration and conflict - specifically, the weights given to vision and haptics when stimulus size is estimated. The term haptic refers to a perceptual system that combines both input from receptors in the skin and kinesthetic information. Kinesthetic perception is mediated by variations in the movement and position of body parts (e.g., joint position and angle). In the experiments reported here, haptic conditions were manipulated so that kinesthetic information was presented either alone or in conjunction with cutaneous information. We will show that visual information is relied on more heavily than is haptic information in only some conditions (e.g., kinesthetic-only condition).

Although sensory integration and conflict have been of interest for many years (Phillips, Egan, \& Perry, 2009; Rock \& Victor, 1964), it is only recently that the technology has become available to more systematically pit one sense against another. New ways of delivering discrepant stimuli avoid some of the pitfalls associated with earlier work. Research employing distorting prisms, lenses, and similar devices often suffered from the criticism that an exploring hand or finger was distorted along with the stimulus. For example, Easton and Moran's (1978) subjects viewed their own finger running along a rod. In the condition in which a distorting lens was used to make the rod appear curved, the subjects' finger also appeared distorted. McDonnell and Duffett (1972) wondered whether a distorted finger in such research would encourage attention to the visual modality and, perhaps, attenuate the tactual information.
Prisms have also been used to blur the visual field. In their experiments, Fishkin, Pishkin, and Stahl (1975) asked subjects to haptically match the orientation of an objectively vertical rod. These authors blurred the visual field, making the position of the rod less precise. Since they were testing the modality appropriateness hypothesis, it was speculated that haptics would play a greater role, relative to vision. Modality appropriateness suggests that when two senses supply incongruent input about an event or an object, a unitary impression is created in favor of the input from the modality with the greatest precision with respect to that particular information (Ernst \& Bülthoff, 2004; Spence \& Walton, 2005; Welch \& Warren, 1980). Haptics was not relied on more heavily than vision, but this may have been due to inadequate attempts to match the stimuli.

Loomis, Klatzky, and Lederman (1991) considered several important ways in which visual inputs are favored over their corresponding haptic renditions and noted the need to match modal inputs as much as is possible. Soto-Faraco and Deco (2009) argued that the similarities between hearing and touch (i.e., functional properties, sensitivity to a certain type of physical energy, and the possibility that they share an evolutionary origin) present a promising approach to systematically studying sensory integration. It is somewhat surprising, given the renewed interest in studying sensory dominance, that there have been few attempts to match the information presented to vision and haptics. To address these concerns, we prepared stimuli designed to avoid distortions and to match the experience of the two modalities.

G. H. Van Doorn, george.vandoorn@arts.monash.edu.au 
An interesting aspect of some experiments on sensory discrepancy is the use of a unimodal choice set. Hershberger and Misceo (1996) described an experiment conducted by Klein (1966) in which subjects simultaneously viewed and felt a peg made from two different-sized squares - one presented visually, the other tactually. While visually and tactually examining the peg, the subjects used their free hand to select a haptic match. Although it may have been Klein's intention to draw more attention to the haptic modality, this may have biased the results. Klein's results trended toward haptic dominance, and it may be that people expecting to make a haptic judgment at the conclusion of each trial, either deliberately or inadvertently, rely on the information presented tactually during that trial. Consequently, they may ignore important information present at the conflicting modality-vision, in this case. Although there is some evidence to suggest that the mode to which the choice set is presented is not a crucial factor (e.g., Easton \& Moran, 1978), more recent research indicates that whichever modality is used for exploring comparison stimuli is also the mode that tends to dominate (Hershberger \& Misceo, 1996). This possibility was taken into account in the present research by providing sets of visual and haptic choice stimuli across different experiments, thus testing for effects attributable to the modality used to select choice squares.

Another factor may cast doubt on previous experiments that have purported to demonstrate dominance. This has to do with the lack of a baseline measure of performance prior to testing in conditions involving conflict. Some experimenters have included tests of baseline performance but have, nevertheless, failed to assess the extent to which one modality has had an influence on the other. In order to assess the influence of one modality on another when both have the potential to offer information, it is necessary to assess how much information is delivered by each modality alone. The intersensory bias literature (see Soto-Faraco, Spence, \& Kingstone, 2004, for example) typically has proposed a model of integration, rather than capture, in which information in one modality is made to conform to information in another. However, few authors have actually determined the extent of reciprocal influence. For example, McDonnell and Duffett (1972) suggested that size estimates made by subjects using touch and vision were intermediate between those made by subjects using vision alone and others using touch alone. Specifically, visionalone, touch-alone, and bimodal inspection of stimulus squares resulted in estimates of $2.46,1.53$, and 1.84 in., respectively. McDonnell and Duffett suggested that these findings make it more probable that bimodal trials bore greater resemblance to the touch-alone trials than to the vision-alone trials. Although a value of 1.84 in. was not significantly different from $1.53 \mathrm{in}$. at the .01 level used by these authors, it does appear that vision had some influence on the haptic experience. Indeed, the mean size judgments of $2.46,1.53$, and 1.84 in. were all found to be significantly different from one another at the .05 level. It seems inappropriate to adopt the .01 level of significance if the motive for doing so was to be able to claim that the bimodal mean was closer to the touch-alone mean. McDonnell and Duffett's study appears to be one in which there was an opportunity to consider reciprocal effects (vision on touch and touch on vision), rather than attempt to establish dominance of one modality over the other.

Given the point that the influence of one sense (i.e., vision) on another (i.e., haptics) can be measured only against unimodal baseline data, in the experiments reported here, the accuracy with which square sizes were judged using vision alone or haptics alone was not of particular interest. Instead, these unimodal performance measures served as baselines against which bimodal performance could be compared. If visual judgments in unimodal and bimodal conditions are equally accurate, there is no evidence of haptic influence. Similarly, there is no evidence of visual influence in bimodal stimulus presentations if haptic performance is the same in unimodal and bimodal conditions. However, any departure from these extreme "no-influence" possibilities represents the opportunity to measure reciprocal effects of vision on haptics or haptics on vision.

\section{BIMODAL VISUAL AND KINESTHETIC EFFECTS ON SIZE JUDGMENTS: DO MODALITY INFLUENCES DIFFER?}

\section{Experiment 1 Passive Exploration/Visual Choice Set}

In Experiment 1, vision was restricted to a moving, single point of reference to approximate the haptic experience resulting from a single point of contact. In Experiments $1-4$, haptic input was predominantly kinesthetic but, arguably, not completely without tactile information (i.e., shear forces and pressure changes felt at the fingertip when the stylus came into "contact" with the square pathway). It was expected that vision would dominate touch, as has been reported in studies in which visual input was less closely matched to tactile input (e.g., Rock \& Victor, 1964).

\section{Method}

Subjects. The mean age of the subjects was 25.3 years $(S D=$ 7.0 years; range, $18-41$ years), and 7 of the 10 participants were female.

Apparatus. The visual stimulus was a cone-shaped cursor following a square pathway on a CRT monitor. The tip of the cursor always pointed toward the center of the square, irrespective of the side of the square it traveled along (i.e., the cursor was always at a $90^{\circ}$ angle relative to the side it was touching). The square pathway was presented haptically with a Desktop Version 3.1 force-feedback Phantom (SensAble Technologies Inc.). This Phantom is capable of delivering $9 \mathrm{~N}$ of force, but most stimuli were presented at approximately $5 \mathrm{~N}$.

The monitor was placed centrally in front of each subject, with the Phantom slightly off to the right. A partition extending back to the subject's shoulder and forward beyond the Phantom and monitor was placed between these devices to prevent the subjects from seeing their hand while holding the Phantom's stylus (see Figure 1).

Three square pathways were presented haptically and/or visually, measuring $2.75,3.00$, and $3.25 \mathrm{~cm}$ on the side. In pilot studies, the three stimuli were found to be the minimum number necessary to 
establish an effect. The $0.25-\mathrm{cm}$ size difference between stimuli was also derived from pilot research seeking to minimize floor and ceiling effects. If the task was too easy, so that size differences were easily and reliably detected unimodally, effects of one modality on the other would be hard to detect. For example, during bimodal exploration, the belief that inputs were coming from a single object could be lost or reduced to the point that conflict between modalities would not exist. Accordingly, square sizes were chosen such that detectability of size differences was close to threshold and the influence of one modality on the other might be measurable.

The sizes of the visual and haptic squares could be independently manipulated so that, for example, the pathway taken by the Phantom was $2.75 \mathrm{~cm}$ but the visual counterpart shown on the screen was $3.00 \mathrm{~cm}$. Thus, 15 trials were established: 3 in which the cursor was seen on the screen without a haptic counterpart, 3 haptic-only presentations, and 9 bimodal trials in which the visual and haptic stimuli were presented together. On 6 of the bimodal trials, the visual and haptic sizes of the squares differed, and on 3 trials, they did not.

The subjects were not told that the stimuli were, or could be, set to conflict. Indeed, to strengthen the impression that the multisensory input originated from a single square, cursor movement was calibrated proportionately to the stylus motion, so that the square's corners were reached at the same time regardless of the distance between those corners; thus, cursor speed was manipulated. More specifically, it took $10 \mathrm{sec}$ to move once around the square pathway. Although cursor speed varied as a function of square size, this variation was constant across levels of the size variable.

The visual choice set consisted of cardboard squares numbered 1 through 11, mounted on a wall, at head height, on the subject's lefthand side. These squares increased in increments of $0.25 \mathrm{~cm}$ from 1.75 to $4.25 \mathrm{~cm}$.

Procedure. The subjects observed a cursor travel around a practice square and were passively guided, by the Phantom, around it. Instructions were given verbally by the experimenter. The size of the practice square was visually and haptically $4 \mathrm{~cm}$ (i.e., larger than any stimulus presented during the experimental trials).

The subjects were told that the experiment concerned the ability to accurately assess the size of squares traced out by the cursor and/ or Phantom. The instructions notified the subjects that the size of the

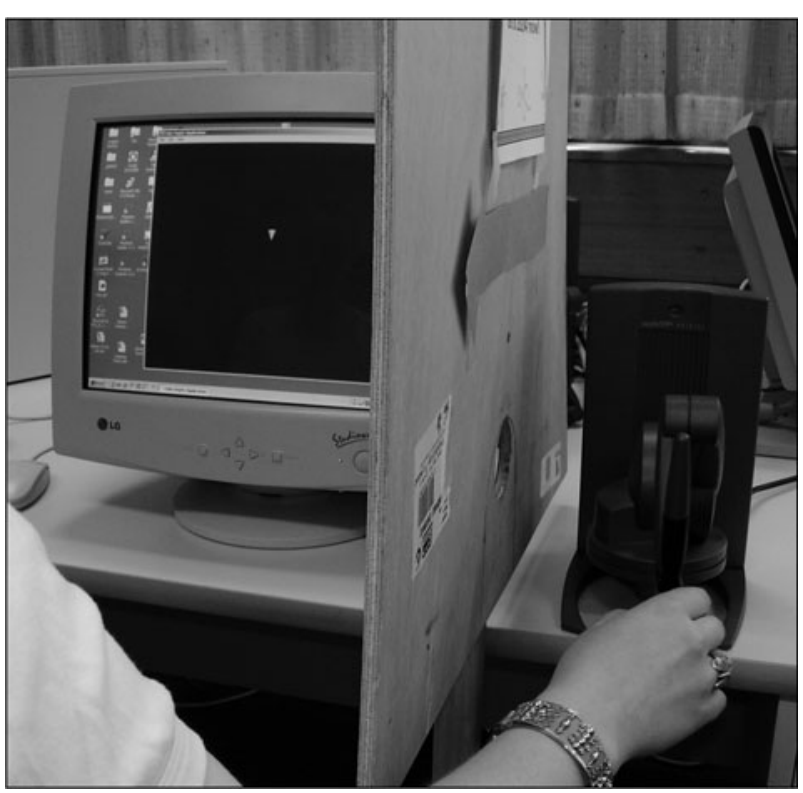

Figure 1. The computer screen, visible cursor, partition, and Phantom. square pathway might change across trials. However, the subjects were not informed that the visual and haptic stimulus size could be different (conflicting) within experimental trials. No feedback was provided until the end of the experiment.

The experiment proper consisted of three conditions: a visionalone condition with only a visible cursor traveling around the border of an invisible square on a CRT monitor, a haptic-alone condition in which the subjects were passively guided around a square pathway with the CRT monitor "blacked out," and a bimodal condition in which both forms of information were present and were either matched (for three of the nine trials) or of different sizes (for six of the nine trials). On the bimodal trials, each size of the three visual stimuli was paired with each of the three sizes of haptic stimuli.

The presentation order of modality conditions and sizes was randomized for each subject. However, the subjects were told which experimental condition to expect on the next trial (i.e., haptic alone, visual alone, or bimodal). The subjects were allowed to explore each stimulus for as long as they liked (i.e., the number of visual and/or haptic laps of the squares was not limited) and were told that when they believed that they had sufficiently explored the stimulus, they were to look at the choice set and make a selection. The subjects were not required to select a match during the practice trial but were shown the choice set during instruction. The subjects could not see the monitor and the choice squares simultaneously and did not view the choice set until they elected to stop viewing/feeling the stimulus. In the haptic-alone and bimodal conditions, they were required to remove their hand from the Phantom before making a judgment. The subjects selected the square from the choice set that they believed matched the size of the pathway taken by the cursor and/or Phantom. They were required to select a match by saying the number (e.g., 7) that corresponded to the square in the display. The subjects were seated an equal distance from the screen and choice set $(\sim 1 \mathrm{~m})$. The experiment lasted approximately $15 \mathrm{~min}$, with the subjects exploring each stimulus or stimulus pair once. The stimuli were typically explored for around $40 \mathrm{sec}$ each.

Design and Statistical analysis. It is important to determine the extent to which one modality has an influence on the other. To achieve this, a two-step analysis was used.

Unimodal baseline data were determined by subtracting the size of the square traced out by the cursor in the vision-alone and hapticalone conditions from the size of the square selected from the choice set. These error scores provided baseline measures of unimodal visual and haptic accuracy.

Experimental data were analyzed using a repeated measures ANOVA on modality of influence (two levels) and size differences (nine levels). The effects of the size differences variable were not, in themselves, of interest with respect to the main question (i.e., to what extent does one modality influence the other?), but the variable existed as a consequence of the need to create size discrepancies. Also, analysis of the size differences factor reduced overall error by removing from it the variance attributable to this factor.

The errors made in the vision-alone trials were subtracted from the vision error scores in the bimodal condition, since any difference between vision-alone errors and bimodal vision errors should be due to the presence of haptics in bimodal vision errors, but not in vision-alone errors (see Table 1). This yielded a measure of the effect of haptics on vision (H-on-V). Similarly, the influence of vision on haptics (V-on-H) was calculated as the amount of error on haptic-alone trials subtracted from haptic errors in the bimodal condition. The modality of influence factor was thus a reflection of possible differences between $\mathrm{V}-\mathrm{on}-\mathrm{H}$ scores and $\mathrm{H}-\mathrm{on}-\mathrm{V}$ scores. For example, suppose that vision-alone errors and bimodal vision errors did not differ. This would imply that the haptic input (present in the bimodal condition) had no influence whatsoever on visual judgments. This is so because if there had been some haptic influence in the bimodal condition, the bimodal vision error would be expected to differ from error in the vision-alone condition. Now suppose that haptic-alone error was found to be very different from 
Table 1

Procedure for Determining Modality of Influence Data

\begin{tabular}{ll}
\hline Effect of haptics on vision & $=$ bimodal vision error - vision-alone error \\
where bimodal vision error & $=$ size of square chosen in bimodal vision-haptic presentation - size of square visually presented \\
and vision-alone error & $=$ size of square chosen in unimodal vision condition - size of square visually presented \\
Effect of vision on haptics & $=$ bimodal haptic error - haptic-alone error \\
where bimodal haptic error & $=$ size of square chosen in bimodal vision-haptic presentation - size of square haptically presented \\
and haptic-alone error & $=$ size of square chosen in unimodal haptic condition - size of square haptically presented
\end{tabular}

bimodal haptic error and that this bimodal haptic error was the same as the error for bimodal vision (which we supposed earlier did not differ from vision-alone error). This would be evidence of a significant visual influence on haptics but no influence whatsoever of $\mathrm{H}$-on-V. It would suggest that vision completely captured haptics. If haptics has no effect on vision judgments, $\mathrm{H}$-on-V will be zero. If vision has no influence on haptic judgments, V-on-H scores will be zero. The extent of the departure from these extreme cases reveals the relative influence of $\mathrm{V}-\mathrm{on}-\mathrm{H}$ and $\mathrm{H}-\mathrm{on}-\mathrm{V}$ in the bimodal condition.

\section{Results}

The subjects were less accurate using vision alone $\left(M_{\text {error }}=0.23 \mathrm{~cm}, S D_{\text {error }}=0.39 \mathrm{~cm}\right)$ than they were using haptics alone $\left(M_{\text {error }}=-0.11 \mathrm{~cm}, S D_{\text {error }}=0.44 \mathrm{~cm}\right)$. However, a 2 (modality of influence) $\times 9$ (size differences) repeated measures ANOVA on size of errors showed that the addition of a visual stimulus to haptics shifted subjects' impression of size further away from their hapticalone estimates $\left(M_{\text {error }}=0.28 \mathrm{~cm}, S D_{\text {error }}=0.31 \mathrm{~cm}\right)$ than did adding a haptic stimulus to estimates made with vision alone $\left(M_{\text {error }}=-0.06 \mathrm{~cm}, S D_{\text {error }}=0.26 \mathrm{~cm}\right)[F(1,9)=$ 9.77, $p<.05$ ]. Figure 2 shows that $\mathrm{H}-\mathrm{on}-\mathrm{V}$ is closer to zero than is $\mathrm{V}$-on-H; thus, vision appeared to have the greater influence on bimodal size perception.
The size of errors varied across the nine size differences $[F(8,72)=2.86, p<.01]$. This significant main effect failed to show any discernible pattern. For example, a Scheffé planned-contrasts analysis showed no significant difference between errors when visual and haptic square sizes were matched (e.g., vision $=2.75 \mathrm{~cm}$, haptics $=$ $2.75 \mathrm{~cm}$ ) and errors when stimulus sizes were discrepant (e.g., vision $=2.75 \mathrm{~cm}$, haptics $=3.25 \mathrm{~cm}$ ). Since there was no interest in the size differences factor per se, further post hoc analysis was not conducted to determine which levels were significantly different from one another. The interaction between size differences and modality of influence did not reach statistical significance.

\section{Discussion}

Felt size was affected by visual information more than seen size was affected by what was felt. Thus, when input to the two modalities was controlled, visual dominance remained. This occurred even though estimates based only on visual information were significantly less accurate than those based only on haptic input — assessed using a pairedsamples $t$ test on modality (vision alone vs. haptic alone).

Visual square size was presented in a manner analogous to that experienced for haptics: A single point, the

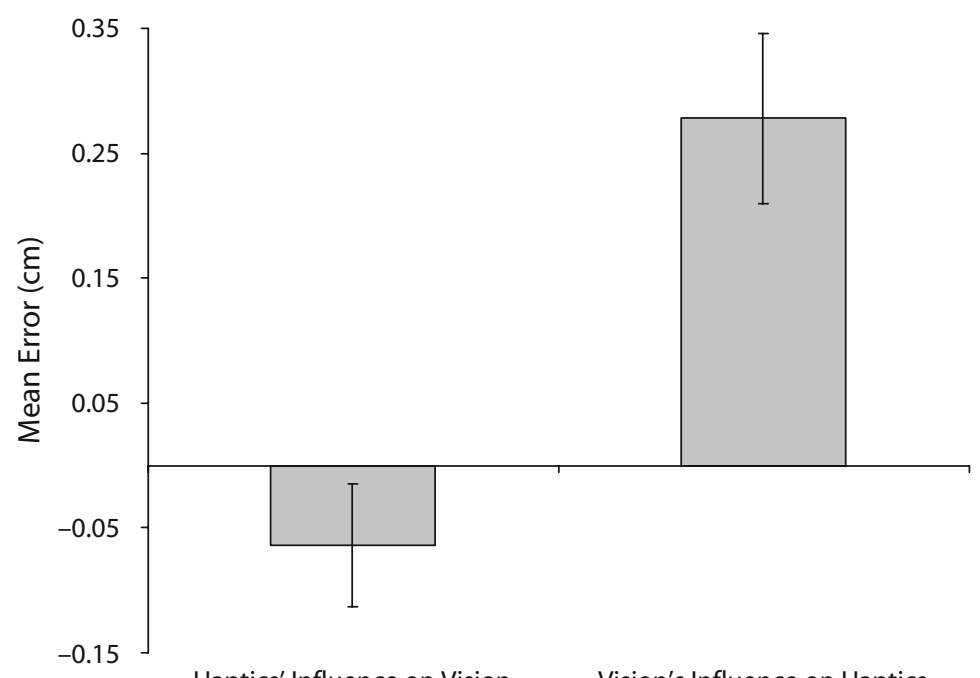

Modality of Influence

Figure 2. Errors in the vision-alone trials were subtracted from visual errors in the bimodal trials to yield the influence of haptics on vision. The influence of vision on haptics was calculated as bimodal haptic error minus haptic-alone error. Data were collapsed across size differences. $S E$ bars are shown. 
cursor, traveled around a pathway and, thus, matched the single haptic point of contact. Consequently, the subjects could not assess the global features of a visible square as they normally would (Lakatos \& Marks, 1999; Rosch, Mervis, Grey, Johnson, \& Boyes-Braem, 1976). This unusual method of presentation may have posed particular difficulty for vision, resulting in the decreased level of accuracy when this modality was used (see Loomis et al., 1991, for a similar argument). Dealing with a single point of visual reference, rather than presenting the complete shape simultaneously, may also have required the subjects to direct a good deal of attention toward the visual modality in the bimodal condition, to compensate for a lack of adequate sensory input. Although devoting more attention to the visual modality would not increase the available information, attending to properties of stimuli can enhance performance on tasks (Motter, 1993).

It is recognized that speed of manual exploration has an influence on haptic estimates of size (e.g., Hollins \& Goble, 1988). In an attempt to address this issue, subjects were allowed to explore each stimulus for as long as they wanted. Kirkpatrick and Douglas (2002) suggested that it takes $22 \mathrm{sec}$ to identify an object, using a Phantom. The subjects here generally explored each square for about $40 \mathrm{sec}$. Given that unimodal haptic estimates were closer to the actual stimulus size than were unimodal visual estimates, it would appear that the speed of manual exploration was not an issue in this experiment.

The haptic condition was passive in Experiment 1, since the subjects had no control over the uptake of information; the movement of the Phantom stylus was set to a predetermined speed and direction of movement. Although the question of which of active and passive haptic exploration is superior is not yet settled (see Symmons, Richardson, \& Wuillemin, 2004), it is possible that active haptic control will increase reliance on haptic information in the bimodal task. Welch, Widawski, Harrington, and Warren (1979) suggested that visual dominance is facilitated by degrading proprioception and kinesthesis. They argued that passive movements play a role in this degradation. Experiment 2 tested the possibility that since active haptic exploration may be better than passive, haptic information might be more heavily relied on if it is gained actively.

\section{Experiment 2 Active Exploration/Visual Choice Set}

The findings of Experiment 1 indicated that information presented visually had more effect on haptic input than vice versa. However, the haptic input was provided in a passively guided mode. Experiment 2 differed from Experiment 1 in that haptic exploration of the square stimuli was active.

\section{Method}

Subjects. Nine female and 2 male students participated in the second experiment. The subjects were between 18 and 41 years of age $(M=26.7, S D=9.4)$.

Materials, Procedure, and Statistical analysis. The stimuli, materials, procedure, and statistical analysis used in Experiment 2 were the same as those used in Experiment 1, with one important exception. In this experiment, the subjects actively moved the Phantom stylus around the stimulus squares. In Experiment 1, the haptic pathway faithfully followed the shape of the square stimuli, since the movement patterns had been prerecorded by the experimenter. Allowing the subjects to control the haptic experience would likely result in occasions in which the subjects unintentionally drifted off the square, particularly at the corners. To allow for this, forces were programmed to attract the Phantom's point of contact to the stimulus - a situation akin to a magnetic effect. This allowed the subjects to "fall off" the square but remain in the vicinity and then be drawn back to it.

\section{Results and Discussion}

The influences of vision and touch on bimodal stimuli were equal. The statistical procedure outlined in Experiment 1 (i.e., a $2 \times 9$ ANOVA on size of errors) did not lead to significant differences in Experiment 2. One interpretation of this outcome is that active touch may have resisted the visual dominance observed in Experiment 1. Attention may have been directed to information presented in the visual modality in Experiment 1 because the visual task was unusual or more difficult than the haptic task. The active haptic mode may have offered better information in Experiment 2. It has been suggested that passive exploration with a Phantom yields weaker object (surface) percepts than does active exploration (Richardson, Symmons, \& Wuillemin, 2006), so that the square may have seemed more "real" when explored actively and less prone to visual dominance.

In both Experiments 1 and 2, the sets from which the subjects selected squares were visually presented. Hershberger and Misceo (1996) have suggested that the dominant modality tends to correspond "to the type of comparison stimuli used by the observers to render their size estimates" (p. 1130). Experiments 3 and 4 were designed to test for effects of the modality of choice set.

\section{Experiments 3 and 4 \\ Passive Exploration/Haptic Choice Set and Active Exploration/Haptic Choice Set}

In these experiments, haptic choice sets were used in place of the visual choice sets employed in Experiments 1 and 2. The hypothesis of interest was that a haptic choice set may favor attention to that modality, with a further reduction in vision's influence from that observed in Experiments 1 and 2 .

\section{Method}

Subjects. The 11 participants in Experiment 3 had a mean age of 24.0 years ( $S D=7.5$; range, $18-44$ years), and 9 of them were women. Experiment 4 involved 10 participants with a mean age of 27.2 years $(S D=10.7$; range, $18-45$ years), 9 of whom were women.

Materials, Procedure, and Statistical analysis. In Experiments 3 and 4, the choice set consisted of eleven 6-mm-thick, square pieces of Masonite glued to a board and arranged in a horizontal row from largest square to smallest. The stimulus set was kept beneath a vision-occluding partition immediately in front of the subjects. The subjects were told that when they had sufficiently explored the stimuli, they were to remove their hand from the Phantom and reach under the occluding partition to select a square from the comparison set. They generally used all fingers to select a likely match, but be- 
fore making a final decision, they often traced around the outside of that square with one finger. The CRT monitor was screened so that they could not see the stimuli while making their choice.

\section{Results}

Experiment 3. The $2 \times 9$ repeated measures ANOVA on the error data failed to produce significant main effects in Experiment 3. The only significant finding was the interaction between modality of influence and size differences (i.e., errors in the bimodal trials minus errors in the alone trials) $[F(8,80)=3.47, p<.01]$. The interaction failed to show any discernible pattern. As such, conclusions about the differences in errors among the levels of modality of influence must be made separately at each level of size differences.

Experiment 4. The statistical procedure outlined in Experiment 1 failed to lead to significant effects in Experiment 4.

\section{Discussion}

The findings of Experiments 3 and 4 suggest that the mode of the comparison set had no effect and that vision and haptics had an equal influence on bimodal size estimations, so far as error scores were concerned. The explanation may be that attention distributed between vision and touch leaves both unable to bias or capture the other.

Although using a probe to haptically explore squares is useful in matching the visual and haptic experiences, it is recognized that limiting the amount of information made available to haptics to a single point substantially weakens performance in identification tasks (see Jansson et al., 1999; Kirkpatrick \& Douglas, 2002). Jansson et al. have further suggested that this may be particularly true for novices, a problem directly relevant to the present set of experiments. In an attempt to provide enhanced haptic information, Experiments 5 and 6 assessed the effects of adding tactile information to the kinesthetic information available in Experiments 1-4 while maintaining the equality of visual and haptic information.

\section{BIMODAL VISUAL AND KINESTHETIC EFFECTS ON SIZE JUDGMENTS: DO MODALITY INFLUENCES DIFFER WHEN TOUCH IS ADDED TO KINESTHESIS?}

\section{Experiment 5 \\ Passive Exploration Including Touch/Visual Choice Set}

It is possible that adding tactile information would result in more attentional resources being devoted to the haptic qualities of the stimuli. If there are errors in vision or touch in these conditions, salience of the tactilekinesthetic information could influence vision in bimodal conditions.

\section{Method}

Subjects. The 10 subjects in Experiment 5 had a mean age of 31.1 years $(S D=11.9$; range, $18-46$ years $)$, and 7 of them were women.
Apparatus and Materials. The visual stimulus consisted of a small portion of line $(\sim 1 \mathrm{~cm})$ that traced out a square-shaped pathway, like watching a short snake travel around an invisible square, on a CRT monitor.

The three invisible stimulus squares had 2.75-, 3.00-, and 3.25-cm side measurements. The software used to generate the lines and squares allowed independent manipulation of visual and haptic stimuli (Richardson, Symmons, Wuillemin, \& Van Doorn, 2005).

Haptic presentation of the squares was achieved using the tactile display system (TDS; Richardson, Symmons, \& Accardi, 2000). It included a plastic cradle in which a subject's index finger was lightly gripped, leaving the finger pad free to explore raised-line stimuli. The cradle assembly allowed the subjects to explore the $x$ - and $y$-axes via self-generated movement or by being passively guided by the TDS, which had motors that could drive the cradle within a $10 \times 10 \mathrm{~cm}$ area (see Figure 3 ).

The experimenter actively explored raised-line squares, and the direction and speed of the experimenter's movement were electronically logged by the TDS's optical encoders. These exploration patterns provided the time and movement constraints of each passive trial. The prerecorded movements of the experimenter were replayed to the subjects to guide their index finger around the raised-line squares. To reinforce the subjects' impression that the bimodal stimuli originated from a single square, the movement of the visible line was calibrated so that it arrived at the square's corners at the same time as the TDS guided the subjects' fingertip to the corresponding haptic square's corners.

The computer screen was placed centrally in front of the subjects with the TDS beneath it. A partition was placed between the TDS and the subjects to prevent a direct view of their exploring hand. The 11 visual choice squares used in Experiments 1 and 2 were also used here.

Procedure. The procedure was similar to that in Experiments 1-4. However, the visual stimulus was a short line $(\sim 1 \mathrm{~cm})$, rather than a cursor, that traced out a square-shaped pathway, and the haptic condition included cutaneous contact via a raised line, in addition to kinesthetic information. The visible section of line mimicked the section of raised line felt at the fingertip.

In the haptic-alone and bimodal conditions, the subjects stood facing the TDS, placed their right index finger into the TDS cradle, and made contact with the raised-line surface. The finger was

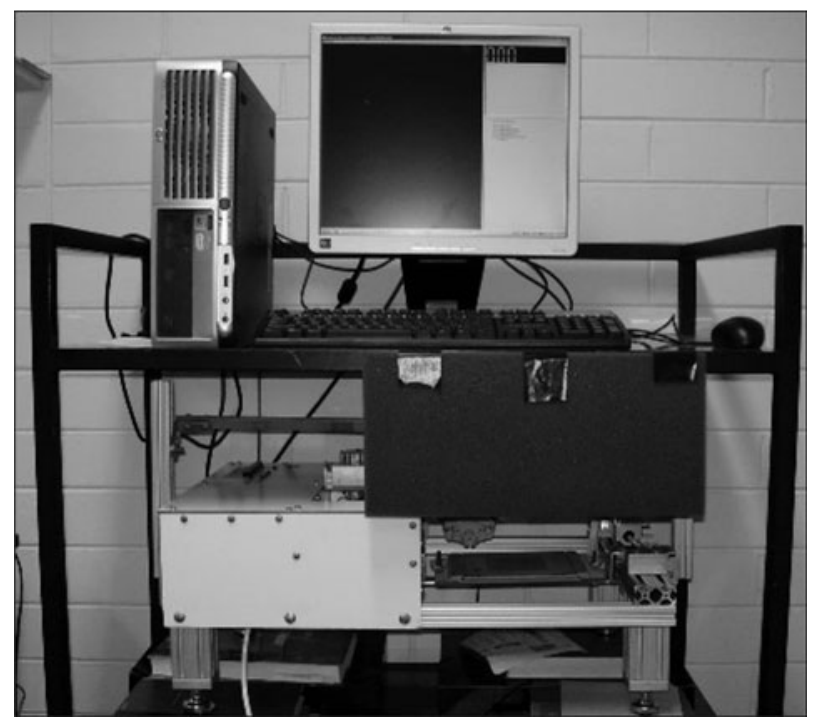

Figure 3. The computer screen, partition, and tactile display system. 
moved progressively and systematically around the raised-line drawing for $30 \mathrm{sec}$.

The experiment involved three conditions: a vision-alone condition with only a visible line traveling around an invisible square, a haptic-alone condition in which the subjects were passively guided around a raised-line square, and a bimodal condition in which both forms of input were present.

\section{Results}

Using vision alone, the subjects were less accurate $\left(M_{\text {error }}=-0.63 \mathrm{~cm}, S D_{\text {error }}=0.29 \mathrm{~cm}\right)$ than they were using haptics alone ( $M_{\text {error }}=0.08 \mathrm{~cm}, S D_{\text {error }}=0.40 \mathrm{~cm}$ ). Adding a haptic stimulus to visual information shifted the subjects' impression of size further away from their visionalone estimates $\left(M_{\text {error }}=0.73 \mathrm{~cm}, S D_{\text {error }}=0.25 \mathrm{~cm}\right)$ than did adding a visual stimulus to estimates made in hapticalone conditions $\left(M_{\text {error }}=0.03 \mathrm{~cm}, S D_{\text {error }}=0.41 \mathrm{~cm}\right)$ $[F(1,9)=16.82, p<.01]$. Figure 4 shows that since $\mathrm{V}$-on-H is closer to zero than $\mathrm{H}-\mathrm{on}-\mathrm{V}$, haptic information, when both modalities are used together, "dragged" the visual experience away from what it was during the vision-alone condition. An analysis of the error data did not reveal a significant main effect of size differences or a significant interaction between it and the modality-ofinfluence factor.

\section{Discussion}

Visual input was more affected by concomitant haptic information than felt size was affected by vision. The addition of cutaneous information was apparently effective in establishing haptic dominance.

Again, judgments in the haptic-alone condition were more accurate than those made using vision alone. That is, the subjects could more accurately match a square from the set of visual standards to one felt with the TDS than they could to one traced out on a monitor. Lakatos and Marks (1999) suggested that this is to be expected. People use touch to build up an impression of an object from sequentially presented information. Relatively small surface areas must be integrated over time and in succession (Hadjikhani \& Roland, 1998; Lakatos \& Marks, 1999). When using vision, on the other hand, we often assess the global features of an object before looking for finer detail (Lakatos \& Marks, 1999; Rosch et al., 1976). Due to the reliable haptic information, attention may have been directed toward haptics in the bimodal conditions.

\section{Experiment 6 Passive Exploration Including Touch/Tactile Choice Set}

If cutaneous information is a key variable in hapticvisual conflict, haptic influence should also be predominant if the choice set is haptic. This hypothesis was explored in Experiment 6.

\section{Method}

Subjects. The 10 subjects in Experiment 6 had a mean age of 31.5 years $(S D=12.4$; range, $18-50$ years $)$, and 7 of them were women.

Materials, Procedure, and Statistical analysis. The stimuli, procedure, materials, and statistical analyses used in Experiment 6 were the same as those used in Experiment 5. However, the choice set squares were haptic, as in Experiments 3 and 4.

\section{Results}

A 2 (modality of influence) $\times 9$ (size differences) repeated measures ANOVA on error data showed a significant main effect for the modality-of-influence factor

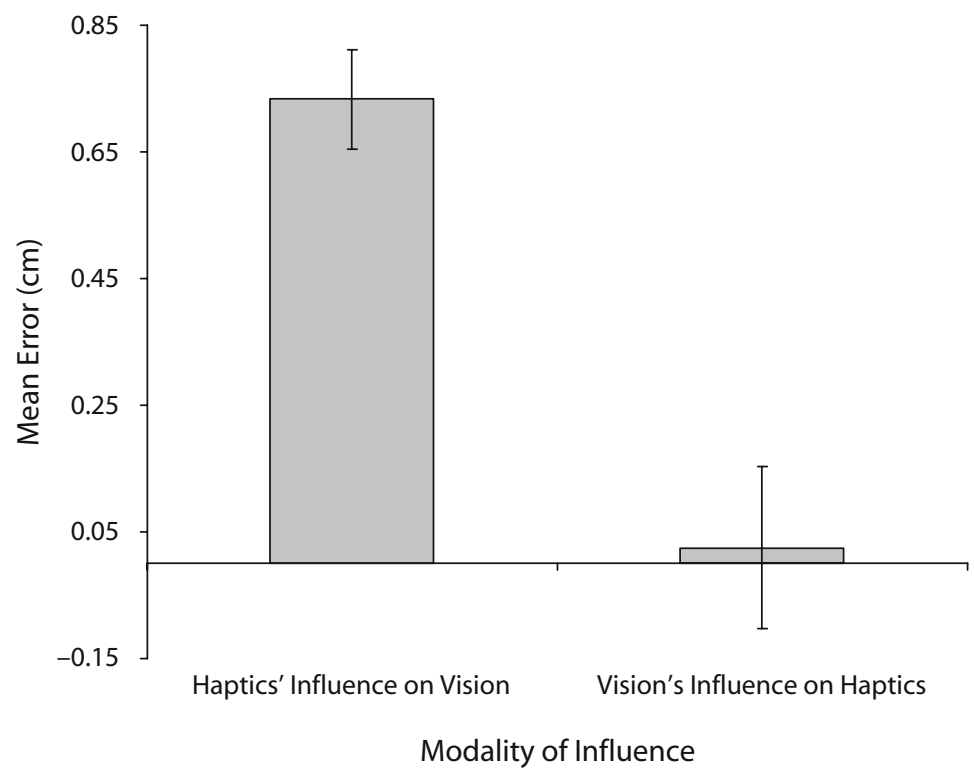

Figure 4. Errors in the haptics-alone trials were subtracted from haptic errors in the bimodal condition to yield the effect of vision on haptics. The effect of haptics on vision was calculated as bimodal visual error minus vision-alone error. Data were collapsed across size differences. $S E$ bars are shown. 
$[F(1,9)=14.82, p<.01]$. Figure 5 shows that the addition of a haptic stimulus to the vision stimulus shifted the subjects' impression of size further away from their vision-alone estimates $\left(M_{\text {error }}=-0.59 \mathrm{~cm}, S D_{\text {error }}=\right.$ $0.41 \mathrm{~cm})$ than did adding a visual stimulus to estimates made with haptics $\left(M_{\text {error }}=0.05 \mathrm{~cm}, S D_{\text {error }}=0.28 \mathrm{~cm}\right)$. The main effect of the second variable (size differences) was not significant.

The interaction between modality of influence and size differences was significant $[F(8,72)=3.51, p<.01]$. The interaction indicates that the difference between errors in $\mathrm{H}-\mathrm{on}-\mathrm{V}$ and $\mathrm{V}-\mathrm{on}-\mathrm{H}$ were significantly larger for size difference $4\left(M_{\text {difference }}=1.10 \mathrm{~cm}\right)$ than for size difference $2\left(M_{\text {difference }}=0.15 \mathrm{~cm}\right)$, since these were the largest and smallest differences, respectively. Other differences may have been significant, but, since the size differences variable emerged as a consequence of the need to include discrepant and congruent trials, these were not of interest, and the data were not examined further. It should be said that even though it is clear that haptics had a greater influence on vision than vice versa, a single conclusion about modality of influence will not hold for all levels of size differences.

\section{Discussion}

In Experiments 5 and 6, cutaneous information was combined with kinesthetic information. Whether the choice set was visual (Experiment 5) or haptic (Experiment 6), the judgments were influenced more by information presented to the haptic modality.

\section{GENERAL DISCUSSION}

Vision can influence judgments made in bimodal conditions (Experiment 1), but haptics can also exert an in- fluence over vision (Experiments 5 and 6), and the two influences can be equal (Experiments 2,3, and 4). The experiment favoring visual superiority pitted vision against passive haptics with no cutaneous input, except shear forces that may have been detectable where the Phantom stylus was gripped. Haptic dominance was established by combining kinesthesis and cutaneous input. The change in influence can be explained by the reliability of information, since active exploration and the addition of cutaneous information boost the impact of haptics.

The experiments more closely match visual and haptic experience than has been the case in previous research, since the visual input is analogous to the haptic one. In Experiments $1-4$, vision was diminished to a single point of reference, a situation that emulates the Phantom's single point of contact with a virtual surface. In Experiment 1, in which haptic exploration was passive, perception of size was dominated by the visual input (see Figure 2). However, against active haptic exploration, vision did not dominate (Experiment 2). In Experiments 3 and 4, subjects expecting to make a haptic judgment were influenced as much by haptics as by vision.

In Experiments 5 and 6, with cutaneous and kinesthetic form information combined, bimodal perception of size was dominated by the haptic input, despite passive conditions of exploration. These results have relevance to hypotheses relating to sensory dominance.

Proponents of the modality appropriateness hypothesis argue that when two senses supply incongruent input about an event or object, a unitary impression is created in favor of the input from the modality with the greatest precision with respect to that particular information (Ernst \& Bülthoff, 2004; Spence \& Walton, 2005; Welch \& Warren, 1980). This hypothesis implies that the degree to which one modality dominates another can be altered by

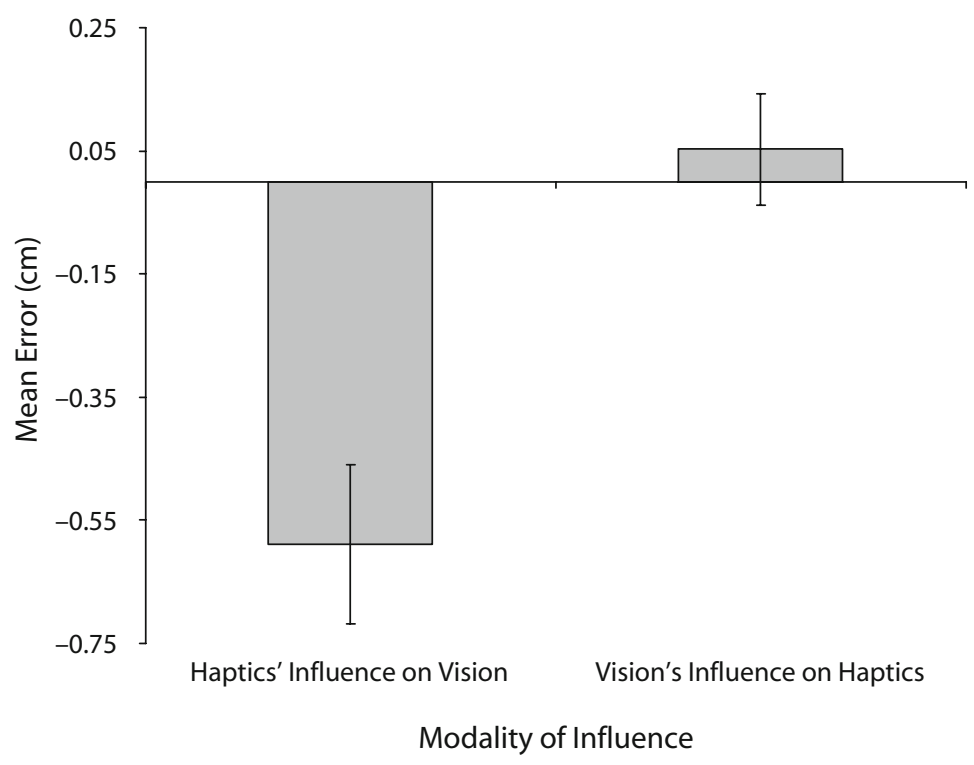

Figure 5. The effect of vision on haptics and haptics on vision. Data were collapsed across the size differences variable. $S E$ bars are shown. 
adjusting the amount of information a modality receives. The findings in Experiments 2 and 3, in conjunction with those from Experiments 5 and 6, provide some supportive evidence for this hypothesis. After the relative amounts of information made available to the haptic modality was manipulated by changing movement type from passive to active (Experiment 2) and choice set from visual to haptic (Experiment 3), the visual dominance observed in Experiment 1 was no longer apparent. More important, by adding cutaneous information to the kinesthetic input, the haptic modality was observed to have a greater influence on vision than vice versa, perhaps because haptics became more accurate and discriminating than vision and, therefore, more appropriate and more relied on.

A consideration that may challenge the role of either modality in the present arrangement is the consequences of directing attention to a particular location, rather than to a particular modality (Spence, 2002). In the present set of experiments, haptic and visual stimuli were presented at disparate locations. It is true that this situation makes it impossible to quantify the contributions of attending to a particular location or to a particular modality (Spence \& Driver, 1997). Although the present findings may be prejudiced by cues from the location of each stimulus, this was unavoidable in the present situation (i.e., the Phantom and TDS are too large to be placed in the same spatial location as a PC screen). If the results of Experiments 5 and 6, for example, were interpreted in a manner similar to that proposed by Bacon and Shaw (1982), it could be argued that although a position difference may have alerted the subjects to the presence of a conflict, estimates of size still reflected more of the haptic, and not the visual, information. Furthermore, Helbig and Ernst (2007) have suggested that visual and haptic shape information can be readily integrated regardless of whether the stimuli emanate from different or coinciding locations. This effect is, however, dependent on the subjects' belief that they are seeing and feeling a single object.

In summary, visual signals have a higher weighting than do haptic inputs in forming an impression of size if there is limited information presented in the haptic modality. But the weighting of the modalities can be equal if information provided to haptics is heightened, as when the condition is active, rather than passive, or when a haptic choice set is used. Haptics can dominate when touch is added to kinesthesis. In addition, integration of bimodal signals takes the form of dominance, rather than absolute capture. That is, dominated inputs are not entirely supplanted by dominant inputs (Soto-Faraco et al., 2004). We used a measure that took into account the effects of the dominated modality on the dominant modality. This allowed a comparison of the effects of vision on haptics with the effects of haptics on vision, given a particular conflict of stimulus size. The results indicated that when stimuli are equated for difficulty, the weighting a modality receives, or the level of dominance it exerts, depends on the amount and type of information provided - a finding that is consistent with the modality appropriateness hypothesis of sensory dominance.

\section{AUTHOR NOTE}

Correspondence concerning this article should be addressed to $\mathrm{G}$. $\mathrm{H}$. Van Doorn, Bionics and Cognitive Science Centre, School of Humanities, Communications, and Social Sciences, Monash University, Gippsland Campus, Churchill, Victoria 3842, Australia (e-mail: george .vandoorn@arts.monash.edu.au).

\section{REFERENCES}

Bacon, J. H., \& SHAw, L. (1982). Effect of conflict awareness on visual dominance. Perceptual \& Motor Skills, 54, 263-267.

Easton, R. D., \& Moran, P. W. (1978). A quantitative confirmation of visual capture of curvature. Journal of General Psychology, 98, 105-112.

Ernst, M. O., \& Bülthoff, H. H. (2004). Merging the senses into a robust percept. Trends in Cognitive Sciences, 8, 162-169.

Fishkin, S. M., Pishkin, V., \& Stahl, M. L. (1975). Factors involved in visual capture. Perceptual \& Motor Skills, 40, 427-434.

HadjikHani, N., \& Roland, P. E. (1998). Cross-modal transfer of information between the tactile and the visual representations in the human brain: A positron emission tomographic study. Journal of Neuroscience, 18, 1072-1084.

Helbig, H. B., \& ERnst, M. O. (2007). Knowledge about a common source can promote visual-haptic integration. Perception, 36, 15231533.

Hershberger, W. A., \& Misceo, G. F. (1996). Touch dominates haptic estimates of discordant visual-haptic size. Perception \& Psychophysics, 58, 1124-1132.

Hollins, M., \& Goble, A. K. (1988). Perception of the length of voluntary movements. Somatosensory Research, 5, 335-348.

Jansson, G., Billberger, K., Petrie, H., Colwell, C., Kornbrot, D., FÄNGER, J., ET AL. (1999). Haptic virtual environments for blind people: Exploratory experiments with two devices. International Journal of Virtual Reality, 4, 10-20.

KirkPatrick, A. E., \& Douglas, S. A. (2002, March). Application-based evaluation of haptic interfaces. In Proceedings of the 10th Symposium on Haptic Interfaces for Virtual Environment and Teleoperator Systems (pp. 32-39). Washington, DC: IEEE Computer Society Press.

KLEIN, R. (1966). A developmental study of perception under conditions of conflicting sensory cues. Dissertation Abstracts, 27, 2162B-2163B.

LAKATOS, S., \& MARKs, L. E. (1999). Haptic form perception: Relative salience of local and global features. Perception \& Psychophysics, 61, 895-908.

Loomis, J. M., Klatzky, R. L., \& Lederman, S. J. (1991). Similarity in tactual and visual picture recognition with limited field of view. Perception, 20, 167-177.

McDonnell, P. M., \& Duffett, J. (1972). Vision and touch: A reconsideration of conflict between the two senses. Canadian Journal of Psychology, 26, 171-180.

Motter, B. C. (1993). Focal attention produces spatially selective processing in visual cortical areas V1, V2, and V4 in the presence of competing stimuli. Journal of Neurophysiology, 70, 909-919.

Phillips, F., Egan, E. J. L., \& Perry, B. N. (2009). Perceptual equivalence between vision and touch is complexity dependent. Acta Psychologica, 132, 259-266.

Richardson, B. L., Symmons, M., \& Accardi, R. (2000). The TDS: A new device for comparing active and passive-guided touch. IEEE Transactions on Rehabilitation Engineering, 8, 414-417.

Richardson, B. L., Symmons, M., \& Wuillemin, D. B. (2006). Passive exploration and static skin might be more useful than previously assumed. In A. Khedder (Ed.), Proceedings of Eurohaptics 2006 (pp. 57-59). Paris: Académie des Technologies.

Richardson, B. L., Symmons, M., Wuillemin, D. B., \& VAn Doorn, G. H. (2005, March). Looking through a fingertip. Poster presented at the World Haptics Conference, Pisa, Italy.

Rock, I., \& Victor, J. (1964). Vision and touch: An experimentally created conflict between the two senses. Science, 143, 594-596.

Rosch, E. H., Mervis, C. B., Grey, W. D., Johnson, D. M., \& BoyesBraem, P. (1976). Basic objects in natural categories. Cognitive Psychology, 8, 382-439. 
Soto-Faraco, S., \& Deco, G. (2009). Multisensory contributions to the perception of vibrotactile events. Behavioural Brain Research, 196, $145-154$.

Soto-Faraco, S., Spence, C., \& Kingstone, A. (2004). Cross-modal dynamic capture: Congruency effects in the perception of motion across sensory modalities. Journal of Experimental Psychology: Human Perception \& Performance, 30, 330-345.

Spence, C. (2002). Multisensory attention and tactile informationprocessing. Behavioural Brain Research, 135, 57-64.

SPENCE, C., \& Driver, J. (1997). On measuring selective attention to an expected sensory modality. Perception \& Psychophysics, 59, 389-403.

Spence, C., \& Walton, M. (2005). On the inability to ignore touch when responding to vision in the crossmodal congruency task. Acta Psychologica, 118, 47-70.
Symmons, M., Richardson, B. L., \& WUillemin, D. B. (2004). Active versus passive touch: Superiority depends more on the task than the mode. In S. Ballesterios \& M. Heller (Eds.), Touch blindness and neuroscience (pp. 179-185). Madrid: Universidad Nacional de Educacion a Distancia.

WeLCH, R. B., \& WARREN, D. H. (1980). Immediate perceptual response to intersensory discrepancy. Psychological Bulletin, 88, 638-667.

Welch, R. B., Widawski, M. H., Harrington, J., \& Warren, D. H. (1979). An examination of the relationship between visual capture and prism adaptation. Perception \& Psychophysics, 25, 126-132.

(Manuscript received September 21, 2009; revision accepted for publication October 30, 2009.) 